\title{
El significado del cuidado enfermero en el contexto de la pandemia COVID-19
}

\author{
Jorge Eduardo Perez-Toríz ; Francisco Javier Báez-Hernández ${ }^{2 *}$; Marcela Flores-Merlo ${ }^{3}$; Vianet Nava- \\ Navarro ${ }^{4}$; Arelia Morales-Nieto ${ }^{5}$; Miguel Ángel Zenteno-López ${ }^{6}$
}

\begin{abstract}
RESUMEN
Introducción: El brote infeccioso provocado por la nueva cepa de coronavirus, ha provocado una pandemia con graves consecuencias sanitarias y sociales, que hacen suponer cambios en los significados que se tiene, de lo que es el cuidado de enfermería. Objetivo: explorar el significado de cuidado que tiene el profesional de enfermería ante la pandemia de SARS-CoV2, en un hospital COVID de la ciudad de Puebla, México. Metodología: se desarrolló un estudio cualitativo descriptivo, basándose en la perspectiva fenomenológica interpretativa. El tamaño de muestra se determinó hasta alcanzar el punto de saturación. Se realizaron entrevistas a profundidad que fueron grabadas y transcritas en su totalidad. Se realizó análisis de contenido donde se identificaron temas emergentes. La interpretación de la información fue mediante el método de Colaizzi. Resultados: se encontró que el significado del cuidado de enfermería, es entendido como una responsabilidad dirigida hacia la familia, el paciente y hacia sí mismo, donde emergen dos tipos de cuidado: complejo y de protección, dentro de un ambiente de incertidumbre, pero que, a partir del afrontamiento de sus miedos, dan como resultado: un aprendizaje y una mayor experiencia. Conclusiones: el cuidado de enfermería durante la pandemia se muestra como un valor que surge de un estado de consciencia, para cumplir con la obligación que tiene con los otros y lo otro, donde se reconfigura su papel en la sociedad.
\end{abstract}

Palabras clave: Atención de Enfermería; Pandemias; Infecciones por Coronavirus; Investigación Cualitativa; Personal de Enfermería en Hospital (DeCS).

${ }^{1}$ Licenciado en Enfermería, Estudiante de Maestría. Hospital de Traumatología y Ortopedia de la Secretaría de Salud del Estado de Puebla, México.

https://orcid.org/0000-0002-8979-5422

2Doctor en Ciencias de Enfermería. Facultad de Enfermería, Benemérita Universidad Autónoma de Puebla. https://orcid.org/0000-0003-4986-6596

${ }^{3}$ Doctora en Educación. Facultad de Enfermería, Benemérita Universidad Autónoma de Puebla. https://orcid.org/0000-0001-8549-3235

${ }^{4}$ Doctora en Ciencias de Enfermería. Facultad de Enfermería, Benemérita Universidad Autónoma de Puebla. https://orcid.org/0000-0002-8670-3510

${ }^{5}$ Doctora en Ciencias de Enfermería. Facultad de Enfermería, Benemérita Universidad Autónoma de Puebla. http://orcid.org/0000-0001-6974-9130

${ }^{6}$ Maestro en Enfermería. Facultad de Enfermería, Benemérita Universidad Autónoma de Puebla. https://orcid.org/0000-0002-0551-0902

*Autor para correspondencia: javier.baez@correo.buap.mx

Recibido: $28 / 10 / 2020$

Aceptado: 30/11/2020 


\title{
The meaning of the nursing care in the context of the COVID-19 pandemic
}

\begin{abstract}
Introduction: The infectious outbreak caused by the new coronavirus strain has caused a pandemic with severe health and social consequences, which make us think changes should be made in the current meanings there are nowadays with respect to nursing care. Objective: Explore the meaning of care that has the nursing professional before the SARS-CoV2 pandemic, in a COVID hospital in the city of Puebla, Mexico. Methodology: A qualitative-descriptive study was developed based on the interpretative phenomenological perspective. The size of the sample was determined up to reach the saturation point. Deep interviews were carried out which were recorded and transcribed in their entirety. A content analysis was carried out where emerging issues were identified. The interpretation of the information was made using the Colaizzi method. Results: A meaning of nursing care was found, which is understood as a responsibility addressed to the family, patient, and oneself, where two types of care emerged, namely, complex care and protection care, within an environment of uncertainty, but, parting from fear facing, the result is learning and more experience. Conclusions: Nursing care during the pandemic is shown as a value that emerges from a consciousness status, to meet the obligation that it has with the others and the other, where its role in society is reconfigured.
\end{abstract}

Key words: Nursing care; Pandemics; Coronavirus infections; Qualitative Research; Nursing Personnel in the Hospital (DeCS) 


\title{
O significado do cuidado de enfermagem no contexto da pandemia COVID-19
}

\begin{abstract}
ABSTRATO
Introdução: O surto infeccioso causado pela nova cepa do coronavírus gerou uma pandemia com graves repercussões sociais e de saúde, o que nos leva a pensar que devem ser feitas mudanças nos significados atuais que existem a respeito da assistência de enfermagem. Objetivo: Explorar o significado do cuidado que tem o profissional de enfermagem diante de pandemia de SARS-CoV2, em um hospital COVID na cidade de Puebla, no México. Metodologia: Estudo qualitativo-descritivo desenvolvido a partir da perspectiva interpretativa fenomenológica. O tamanho da amostra for determinado até atingir o ponto de saturação. Foram realizadas entrevistas em profundidade, gravadas e transcritas na íntegra. Foi realizada uma análise de conteúdo onde foram identificadas questões emergentes. A interpretação das informações foi feita pelo método Colaizzi. Resultados: Foi encontrado um sentido do cuidado de enfermagem, entendido como uma responsabilidade dirigida à família, ao paciente e a si mesmo, onde emergiram dois tipos de cuidado, a saber, cuidado complexo e cuidado protetor, em um ambiente de incertezas, mas a partir do medo enfrentando, o resultado é aprendizado e mais experiência. Conclusões: $O$ cuidado de enfermagem durante a pandemia mostra-se como um valor que emerge de um estado de consciencia, para cumprir a origacao que tem como o outro, onde o seu papel na sociedade é reconfigurado.
\end{abstract}

Palavras-chave: Cuidados de enfermagem; pandemia; infecções por coronavírus; pesquisa qualitativa; pessoal de enfermagem no hospital (DeCS). 


\section{INTRODUCCIÓN}

El brote infeccioso provocado por la nueva cepa de coronavirus (SARS-CoV-2), posteriormente denominada enfermedad de COVID-19, ha provocado una pandemia con graves consecuencias sanitarias y sociales ${ }^{1}{ }^{2}$. En México, esta enfermedad se ha propagado de manera exponencial, generando un gran número de casos y muertes en la población ${ }^{3}$.

En este contexto, el personal de enfermería como elementos clave para la contención (prevención, control y rehabilitación) de esta enfermedad, han sacrificado su propio bienestar, debido entre otras cosas, a la presión asistencial y al miedo a contagiarse que, sumado al conocimiento de la muerte de sus compañeros, han comenzado a causarles una gran presión psicoemocional ${ }^{4}$. Hechos, que hacen suponer cambios en los significados que tienen, acerca de lo que es el cuidado de enfermería.

En este sentido, algunos estudios refieren que el significado de cuidado de enfermería se relaciona con la ocupación y las diversas formas de preocuparse. Donde los profesionales tienden a la impropiedad y a la nivelación de todas las posibilidades de ser. Pero cuando extrapolan la tranquilidad y el ensimismamiento, alcanzan la empatía, el respeto y la indulgencia ${ }^{5}$. Además, han identificado categorías como: acciones de enfermería, sentido de compromiso y preocupación. ${ }^{6}$

Asimismo, en una metasíntesis cualitativa se ha reconocido al cuidado como un proceso dinámico e interactivo, donde se manifiestan intercambios de acciones 0 pensamientos dirigidos hacia un fin específico, que incluyen el respeto e individualización de los seres humanos como entidades con características propias y particulares, el cual va más allá de la enfermedad, partiendo de la esencia humana y la tradición femenina, concluyendo que la verdadera esencia del cuidado radica en el vínculo e interacción humana y por ende, los aspectos técnicos o procedimentales opacan la relevancia social del cuidado y reducen la autonomía disciplinar. ${ }^{7}$
Por lo anterior, si se entiende que el significado que se tenga de un fenómeno condiciona la forma en como es representada la realidad en los sujetos. El cual, puede estar sujeta entre otras cosas a las experiencias vividas por los individuos. ${ }^{8}$ Dicha situación, hace pertinente considerar la relevancia que tiene la fenomenología como método de investigación, para tener un acercamiento a la esencia de las experiencias que tienen los profesionales de Enfermería, en torno al fenómeno del cuidado en situación de pandemia, a partir de sus descripciones, emociones, experiencias, razonamientos o percepciones, a fin de ir construyendo $\mathrm{y} / \mathrm{o}$ redescubriendo sus significados. ${ }^{9}$ Lo anterior, derivado de que la interacción humana: persona-enfermera, es el elemento esencial del cuidado de enfermería y sine qua non de su metaparadigma disciplinario. ${ }^{10}$

Aunado, a que diversos estudios han demostrado que el abordaje acerca de los significados, han permitido fundamentar $y$ desarrollar acciones para mejorar la práctica profesional de enfermería, como la redefinición de conceptos, la construcción de instrumentos, así como la fundamentación de modelos de situación específica de cuidado, que promueven la práctica reflexiva, contextualizada y siempre cambiante en el hacer diario de enfermería. ${ }^{9}, 11$ Resultados que solo son posibles mediante el abordaje de la investigación cualitativa, con enfoque fenomenológico, ya sea descriptivo o interpretativo. Situación que hace evidente la necesidad de comprender las experiencias del profesional de Enfermería, durante esta nueva situación global de salud, a través de formaciones subjetivas, que permitan comprender los significados que tienen los profesionales de enfermería, en torno a su hacer profesional; el cual, actualmente es pilar en la contención de esta emergencia sanitaria mundial.

\section{Objetivo}

Explorar el significado de cuidado que tiene el profesional de enfermería ante la pandemia de SARS-CoV2, en un hospital COVID de la ciudad de Puebla, México. 


\section{METODOLOGÍA}

Se desarrolló un estudio cualitativo descriptivo, basándose en la perspectiva fenomenológica interpretativa, en la que participaron mediante un muestreo intencional, ${ }^{12}$ enfermeras $y$ enfermeros que se encuentran atendiendo a pacientes con SARS-CoV2, adscritos a un hospital COVID de la ciudad de Puebla, México y que de manera voluntaria aceptaron participar en el estudio. El tamaño de la muestra se determinó de forma progresiva durante el transcurso de la investigación, hasta alcanzar la saturación de la información. ${ }^{13}$

Para la recolección de datos se elaboraron preguntas detonadoras, que fueron elaboradas con base a la literatura revisada y a la opinión de expertos. Además, se usó de un diario de campo, debido a que se ha documentado como una herramienta de investigación que permite la reflexión de las observaciones e impresiones de los fenómenos que se estudian ${ }^{14}$. De igual manera, facilita tres procesos formativos: la apropiación del conocimiento, la metacognición, así como la competencia escritural y el sentido crítico $^{15}$, que son importantes en el proceso de la investigación cualitativa.

La investigación, se sometió a revisión por parte de la Secretaria de Investigación y Estudios de Posgrado, de la Facultad de Enfermería de la Benemérita Universidad Autónoma de Puebla, para obtener el registro (SIEP/033/2020) y la autorización correspondiente. Una vez teniendo el aval, se contactaron a los participantes en su lugar de trabajo, donde se les explicó el propósito de la investigación, una vez aceptada su participación, se les realizó una entrevista dentro del hospital, en un lugar que fue acondicionado de manera específica para esta investigación (durante el mes de octubre de 2020). Las entrevistas se elaboraron de forma individual y a profundidad, la cual se caracteriza por adentrarse en la vida del otro para penetrar y detallar en lo trascendente, descifrar y comprender los gustos, miedos, satisfacciones, angustias, zozobras y alegrías significativas que son relevantes para el entrevistado; a fin de interpretar la experiencia del otro. ${ }^{16}$
Las entrevistas fueron grabadas mediante un dispositivo de almacenamiento de voz digital marca Sony, modelo PX470 y transcritas en su totalidad en el programa de Microsoft Word, para lo cual utilizó un método de codificación alfanumérico, con edad y sexo (E1 30 años Mujer, E2 25 años Muer, E3 25 años Hombre, E4 30 años Mujer, E5 31 años Mujer, E6 30 años Mujer, E7 40 años Mujer, E8 25 años Mujer), para la identificación anónima de los participantes. Además, se realizaron anotaciones en el diario de campo antes, durante y después de las entrevistas, a fin de complementar la información observada. Al final, se les agradeció su participación.

Se realizó análisis de contenido ${ }^{13}$ de cada una de las entrevistas, así como de las observaciones obtenidas en el diario de campo. La interpretación de la información se realizó mediante el método de Colaizzi 2, 17, el cual permite estudiar el campo de la conciencia y explorar a las personas en estudio, partiendo de sus experiencias; cuyos pasos están constituidos por siete fases: 1. Definición del fenómeno de interés; 2. Recolección de las descripciones de los participantes; 3 . Lectura y extracción de enunciados significativos, derivados de todas las transcripciones, empleando códigos vivos y sustantivos para la conformación de significados; 4. Organización de los grupos de temas formulados; 5 . Búsqueda de semejanzas o relaciones entre sí, de los grupos de temas; 6 . Elaboración de conceptos para la interpretación de los datos; y 7 . Validación de las interpretaciones elaboradas anteriormente, por los mimos participantes. Asimismo, se consideraron los criterios de credibilidad, transferibilidad, consistencia y confiabilidad de los datos, a fin de asegurar el rigor y la calidad en este tipo de estudios. ${ }^{18}$

Finalmente, el presente estudio se apegó a los principios básicos de bioética y al código de ética de enfermería, así como lo señalado por la Ley General de Datos Personales y el Reglamento de la Ley General de Salud, en referencia a los aspectos éticos de la investigación en seres humanos, además de seguir los protocolos seguridad sanitaria establecidos por el gobierno de México durante esta pandemia. ${ }^{3,} 19$ 


\section{RESULTADOS}

Se entrevistaron un total de ocho profesionales de enfermería ( 1 hombre y 7 mujeres), con las que se llegó a la saturación de los datos. El rango de edad de los participantes fue 25 a 44 años, en su mayoría con más de cinco años de experiencia profesional y cuatro de ellas con especialidad en cuidados intensivos. La duración de las entrevistas oscilo entre los 30 y 45 minutos.

El análisis de contenido, condujo a la identificación de cuatro grupos temáticos (1.experiencias significativas; 2.- emociones; 3.estrategias para afrontar lo que vive en su trabajo; 4.- responsabilidad y conocimiento científico-técnico-humanista ) y nueve subtemas (1.- condición de los sujetos cuidados; 2.- tipo y consecuencias de las experiencias; 3.miedo, frustración y desconocimiento; 4.procesos adaptativos de tipo emocional, física y técnica; 5.- afrontamiento humano: cognitivo, conductual y de protección; 6.- metaparadigma persona; 7.- tipo de cuidado; 8 .- ambiente y 9.- aprendizaje + experiencia), que en su conjunto forman el significado de cuidado (Figura 1).

\section{Tema: experiencias significativas}

En este grupo temático se reconocen dos tipos de experiencias que emergen de las condiciones de juventud y soledad de la muerte de las personas cuidadas. La primera denominada positiva, la cual hace alusión a emociones de satisfacción, así como de acciones que dieron como resultado la buena evolución del individuo. La segunda, conceptualizada como negativa, donde hacen referencia a sentimientos de incertidumbre y a la sobrecarga de trabajo. Ambas experiencias, dieron como resultado un fortalecimiento a sus valores relacionados hacia la vida, así como de su conocimiento técnico-científico-humanista, provocando un aumento en la sobrecarga emocional, que en ocasiones le exige una mayor responsabilidad, que sobre pasa los aspectos profesionales y personales de la enfermera o enfermero que cuida a las personas con SARSCoV2 (Tabla 1).

Tabla 1. Tema: experiencias significativas.

Subtema: condición de los sujetos cuidados.

Código vivo

"Que a veces la edad, entre más jóvenes son más vulnerables y [...] que como enfermeras si te...no había tenido esa experiencia tan fuerte" E1. 30 años Mujer. "pues los pacientes estaban solos[...] y pues los pacientes que fallecieron, pues fallecieron solos, sin ningún apoyo[...]más que el de nosotras" E2. 25 años Mujer.

Subtema: tipo y consecuencias de las experiencias.

Código vivo

"Unas fueron agradables porque el paciente pudo salir del padecimiento o del diagnóstico o diagnósticos que tenía [...] eso era lo más satisfactorio, porque sabes que en conjunto con tus compañeros hiciste un buen trabajo" E5. 31 años Mujer; "fueron en cierta forma desagradables, porque hubo pacientes que tenían probabilidades de poder tener una buena calidad de vida, pero por alguna situación se deterioraban y fallecían, eso te daba mucha incertidumbre" E6. 30 años Mujer; "Algunas ocasiones los pacientes hacían caso omiso y pues fallecieron, pero también ya no estuvo en nuestras manos, porque teníamos trabajo excesivo" E7. 40 años Mujer.

"te deja mucho que aprender y por supuesto valorar la vida [...] porque no sabemos en qué momento puedes dejar de existir[...], sin embargo la experiencia que yo tengo en lo personal [...] seria cuidar la 
salud de todos y la integridad" E2. 25 años Mujer; "Pues me dejaron mucha experiencia, porque nosotros primero desconocíamos lo que era toda la sintomatología de la enfermedad" E7. 40 años Mujer; "Aprendes mucho acerca de la humanidad, ya que vez a los pacientes ponerse mal, piensas en su familia, eres reciproco, tratas hacerlo sentir bien, sientes esa empatía emocional" E8 25 años Mujer; "(Suspiro) experiencias sentimentales, porque siento [...]. que [...]tengo un problema psicológico que más que nada, porque me dolió mucho ver a mucha gente morir y no poder hacer nada" E4 30 años Mujer.

Fuente: elaboración propia de los autores. E=Entrevistado.

\section{Tema: emociones}

En relación con las principales emociones sentidas, cuando entraron por primera vez al área COVID, se identifica el miedo y la frustración, las cuales provocan situaciones de inseguridad, ansiedad, estrés e incertidumbre, originadas por dos tipos de desconocimiento, uno dirigido hacia el saber y el otro hacia el hacer. Pero, que a través de la capacitación refieren una reducción de su miedo, que da como consecuencia, tres procesos adaptativos: Emocional, Física y Motora-Técnica (Tabla 2).

Tabla 2. Tema: emociones.

Subtema: miedo, frustración y desconocimiento.

Código vivo

"Tengo que reconocer que parte de las emociones en un cierto momento, ¿es válido decir que tuve miedo?, si, si tuve miedo[...]i"E3 25 años Hombre; "Frustración porque llega el momento en el que quieres arrancarte todo por querer respirar bien, por querer estar bien, pero pues tienes que aguantar, son todas esas emociones que se te juntan"; E8 25 años Mujer; "no sabía ni que hacer, ni cómo abordar al paciente" E3 25 años Hombre; "por la falta de información, por estar enfrentando algo nuevo, bueno para mí eso era lo que más me preocupaba" E6 30 años Mujer.

Subtema: procesos adaptativos de tipo emocional, física y técnica.

Código vivo

"poco a poco nuestro cuerpo se fue adaptando ... ahorita tuvimos que aprender muchas técnicas y adaptarnos a los equipos que teníamos para poder brindar todos los cuidados de enfermería" E7. 40 años Mujer; "ya los médicos fueron lo que nos aportaron, información de la situación en la que estábamos viviendo y eso fue lo que, bueno a mí en lo particular me tranquilizo" E5 31 años Mujer; "tú tienes que sacar el lado profesional, vencer tus miedos y seguir adelante" E8. 25 años Mujer.

Fuente: elaboración propia de los autores. E=Entrevistado.

\section{Tema: estrategias para afrontar lo que vive en su trabajo}

En cuanto a las estrategias para afrontar lo que vive en su trabajo ante la pandemia se identifican tres tipos de acciones: 1.- las humanas, aquellas que son dirigidas hacia el paciente, 2.- las cognitivo-conductual, enfocadas a sí mismo, y 3.- las protectoras, encaminadas a la familia. Todas estas acciones tienen su origen de una fuente Interna (de sí mimo) y Externa (de su familia), (Tabla 3). 
Tabla 3. Tema: estrategias para afrontar lo que vive en su trabajo.

\begin{tabular}{l}
\hline Subtema: afrontamiento humano cognitivo, conductual y de protección. \\
\hline Código vivo \\
"Trato de ofrecer un poco más de lo que... sea calidad humana con el paciente" E1. 30 años Mujer. "Mi \\
estrategia es información, informarme, leer artículos, leer posibles tratamientos, leer posibles \\
consecuencias" E5. 31 años Mujer. "creo que más que estrategias, sería como las habilidades que uno \\
puede desarrollar con el paciente". E3 25 años Hombre; "Educar a tu familia, para que también ellos \\
prevengan un contagio y pues obviamente prevengas tú también contagiarte junto con ellos". E7 40 \\
años Mujer. \\
"he salido a delante principalmente por mi fuerza mental, saber que pues es para lo que nos \\
formaron" E8 25 años Mujer.; "yo, creo que la familia fue muy importante en esto, bueno gracias a \\
Dios yo cuento con mi familia, con mis hijos, con mi madre" E7 40 años Mujer.
\end{tabular}

Fuente: Elaboración propia de los autores. E=Entrevistado.

\section{Tema: responsabilidad y conocimiento científico-técnico-humanista}

Finalmente, se encontró que el significado del cuidado de enfermería durante la pandemia de COVID 19, emerge de la experiencia, las emociones y las estrategias para afrontar lo que vive en su trabajo, la cual es caracterizada, entendida y asumida como una responsabilidad dirigida hacia el metaparadigma de persona (familia, paciente y hacia sí mismo), donde emergen dos tipos de cuidado: complejo y de protección, dentro de un ambiente de incertidumbre (difícil y desconocido). Pero que, a partir del afrontamiento de sus miedos, dan como resultado: un aprendizaje y una mayor experiencia (Tabla 4).

Tabla 4. Tema: responsabilidad y conocimiento científico-técnico-humanista.

Subtema: metaparadigma de persona

\section{Código vivo}

"Mucha responsabilidad [...] Mucha responsabilidad como enfermera, para mi familia, para mi, para el paciente" E1. 30 años Mujer.

Subtema: tipo de cuidado

\section{Código vivo}

"ya que no es lo mismo cuidar otros tipos de diagnóstico, a algo nuevo que nos estamos enfrentado, es como un poquito más de dedicación, porque se tienen que valorar muchos aspectos [...] digamos, es una forma compleja para poder atender o brindar los cuidados de los diferentes aparatos y sistemas"," E6. 30 años Mujer.

"No debes dejar de hacer nada para, para proteger a todos, a las tres partes importantes de una enfermera, su familia, el paciente y tu propia salud." E2. 25 años Mujer.

Subtema: ambiente

\section{Código vivo}

"porque como tratamiento no tenemos nada, entonces es intentar y siempre pensar positivo en que estás haciendo lo correcto" E5. 31 años Mujer; "Y ante el miedo, ante a la incertidumbre y probablemente a ser contagiado, porque ese es un miedo terrible para todos, tenemos que sacar ante todo nuestra ética, nuestras emociones como enfermeros". E7. 40 años Mujer.

Subtema: Aprendizaje + Experiencia

Código vivo 
"aprendes mucho como del paciente, mucho de los cuidados, cosas que probablemente [...] no nos

enseñan, como tal ...y que quedan como experiencia" E8. 25 años Mujer

Fuente: Elaboración propia de los autores. E=Entrevistado.

Figura 1. Significado del cuidado en el contexto de la pandemia COVID-19.

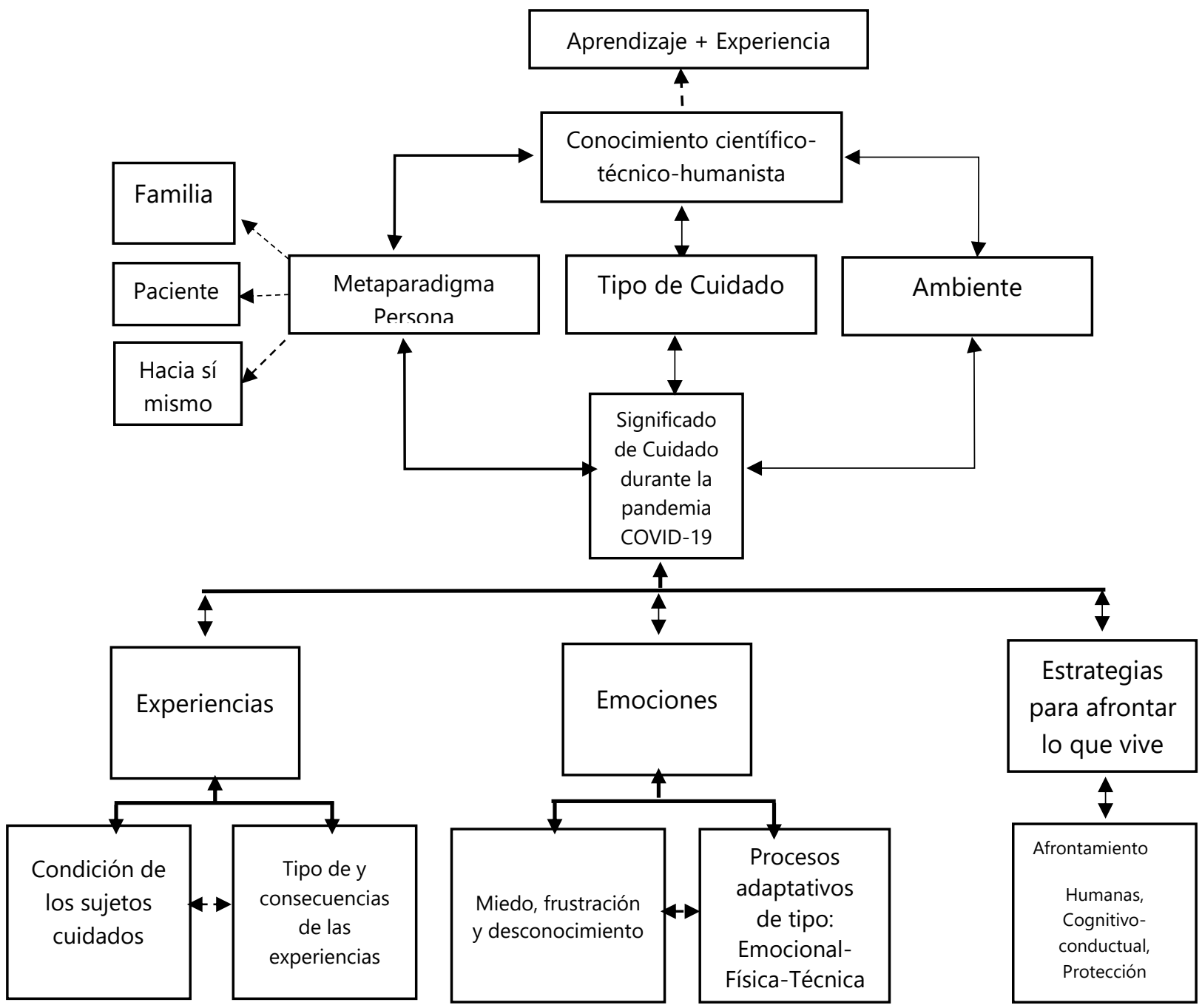

Fuente: Elaboración propia de los autores

\section{DISCUSIÓN}

El cuidado como esencia de la disciplina de Enfermería, es entendido como una responsabilidad, que nace de las experiencias, las emociones y las formas de afrontar lo que viven en su trabajo. Puede interpretarse como un valor implícito de su profesión, que denota no solo la preocupación de los otros o de lo otro, sino también, es la fuerza que lo impulsa adaptarse ante las circunstancias en la que vive. Lo que pudiera coincidir con Peter, 20 al mencionar que algunas inclemencias $y$ perturbaciones durante la vida, afectan el sentido del ser y de la existencia, donde la aceptación, es un reflejo de su salud y funcionalidad, misma que es demostrada en términos de apoyo, paciencia, tolerancia y comprensión; lo anterior, surgido mediante la 
toma de conciencia de sí. Resultado que es concordante con autores españoles quienes refieren, que las enfermeras durante la pandemia de COVID-19, viven situaciones donde coexisten emociones positivas $y$ negativas, generadas por el desconocimiento, el miedo y la falta de insumos, pero que a través de las experiencias positivas logran alcanzar su crecimiento profesional y personal ${ }^{2}$.

Asimismo, se coincide con lo encontrado en un hospital de China, donde los profesionales de enfermería presentaron emociones positivas y negativas entrelazadas durante la pandemia ${ }^{21}$. Situación que hace evidente lo referido por Maturana, 22 al señalar desde el punto de vista biológico, que las emociones son disposiciones corporales dinámicas, que definen $\mathrm{o}$ especifican los distintos dominios de acción y por tanto, determinan sus capacidades de aprendizaje, donde el cuidado, se transforma en un con-vivir con él.

También, se observa una posible re configuración del cuidado, en las que se hacen visibles los patrones del conocimiento de Carper $^{23}$, denotando su carácter científico y humano, el cual le exige no solo el desarrollo de habilidades de razonamiento y análisis, sino también un sentir con y para el otro, que le permita comprender su papel, durante esta epidemia.

En cuanto a la construcción del significado de cuidado, a través de las experiencias de los profesionales de Enfermería que atienden a pacientes con COVID, hacen evidente que las situaciones de enfermería, consideradas como experiencias de vida entre la enfermera y los usuarios, son el depósito del conocimiento enfermero, debido a que permiten reflejar el actuar y la terapéutica del cuidado, confirmando que su significado, reside en el quehacer práctico; por lo que la práctica, es el reflejo del marco epistemológico de enfermería. ${ }^{24,10}$

Además, se pueden distinguir algunos rasgos de las visiones de Enfermería propuestas por Fawcett, 25,26 al pasar por una visión, reactiva, regida por leyes, normas $y$ procedimientos para el manejo adecuado de las personas infectadas. Transitando por una visión de interacción reciproca, donde hace de manifiesto sus valores, creencias y experiencias, donde el cuidado no solo se limita al cuerpo enfermo físicamente, sino que identifica dimensiones psicológicas, sociales y espirituales. Hasta llegar a manifestarse, una visión de simultaneidad, que surge como una forma de interpretar el mundo, caracterizando al cuidado como un fenómeno único, diferente y organizado, que se refleja en un interés genuino por lo que ocurre con el otro. Denotando así, lo razonado por Watson, como un cuidado humanizado con alto compromiso moral.

$\mathrm{Y}$ es desde esta perspectiva de simultaneidad, donde los profesionales de enfermería, pueden ser ubicados para reconocer su proceso transitivo de falta de conocimiento, a la construcción de aprendizajes y experiencias significativas, pasando por posibles procesos adaptativos que simulan lo señalado por Roy, en el que los seres humanos son entes holísticos y trascendentes, en búsqueda de la autorrealización, con la capacidad de tomar decisiones y responsabilidades; en el cual, la adaptación es concebida como un "proceso y resultado" de su conciencia, que eligen para crear una integración humana y ambiental. ${ }^{27}$

Lo anterior expuesto, coincide con el pensamiento fenomenológico y existencialista de Enrique Dussel, el cual concibe al ser humano, como un ser inconcluso e inacabado, que está en la búsqueda y renovación permanente de su ser, hacer y estar con. Situación que concuerda con Arvea ${ }^{28}$, al señalar que los seres humanos que admiten su responsabilidad de su existencia como desafío, se convierten en impulsores de transformación y humanización del mundo.

De igual forma, se coincide con lo afirmado por Swanson, al plantear al cuidado como una forma de relacionarse con el otro, donde emergen sentimientos de compromiso y responsabilidad, en el cual los procesos básicos de conocimiento, estar con, hacer por, posibilitar y el mantenimiento de creencias, hacen evidente la cosmovisión del cuidado. ${ }^{29}$ Que trasciende hacia el otro, en tiempo y espacio, tal y como lo afirma Leonardo Boff, al 
referir a los individuos como seres esencialmente de cuidado, donde la salud de uno depende la salud del otro, dependencia que es asumida conscientemente como solidaridad, en el que pone en descubierto la corresponsabilidad de las acciones, así como la reflexión espiritual sobre el significado y amor a la vida. ${ }^{30}$

\section{CONCLUSIONES}

El cuidado de enfermería durante la pandemia se muestra como un valor que surge de un estado de consciencia, para cumplir con la obligación que tiene con los otros y lo otro, donde se reconfigura su papel en la sociedad. Ante esta situación, hace evidente dos tipos de cuidado: 1.- complejo: dirigido hacia el paciente, y 2.- el de protección: enfocado a su persona y su familia.

También se observan experiencias de cuidado, cargadas de emociones positivas y negativas, que, en voz de los emisores, fortalecieron su sentido hacia la vida, así como su conocimiento técnico-científico y humano.

Finalmente, los resultados del presente estudio permiten tener un acercamiento desde la perspectiva de los profesionales de enfermería que se encuentran viviendo el cuidado durante la pandemia. Contribución que puede sumar al conocimiento disciplinar-científico, que permita hacer visible la importancia del cuidado como esencia de nuestra disciplina.

\section{Limitaciones}

El presente estudio se limitó en un grupo de enfermeras y enfermeros de la ciudad de Puebla, México, que atiende a pacientes diagnosticados con SARS-CoV-2, durante la pandemia de COVID 19, por lo que los resultados no pueden generalizarse, asimismo, solo se abordaron aquellos profesionales que se encontraban con la disposición de tiempo para poder ser entrevistados, guardando siempre los protocolos de seguridad y sana distancia.

\section{CONFLICTO DE INTERESES}

Los autores declaran no tener conflicto de interés.

\section{FINANCIAMIENTO}

Esta investigación no fue financiada por ninguna institución.

\section{AGRADECIMIENTOS}

A los profesionales de Enfermería por compartir sus experiencias.

\section{REFERENCIAS BIBLIOGRÁFICAS}

1. Organización Mundial de la Salud (OMS). Brote de Enfermedad por coronavirus [Internet]. [consultado el 30 de septiembre de 2020]. Disponible en: https://www.who.int/es/emergencies/diseases/ novel-coronavirus-2019.

2. Andreu-Periz D, Ochando-García A, LimónCáceres E. Experiencias de vida y soporte percibido por las enfermeras de las unidades de hemodiálisis hospitalaria durante la pandemia de COVID.19 en España. Enferm Nefrol [Internet]. 2020. [consultado el 27 de octubre de 2020]; Abr-Jun;23(2).148-59. Disponible en: http://dx.doi.org/10.37551/s2254-

28842020022

3. Gobierno de México (2020). Todo sobre el COVID-19 [Internet]. [consultado el 30 de septiembre de 2020]. Disponible en: https://coronavirus.gob.mx/

4. Ferrán MB, Trigo SB. Cuidar al que cuida: El impacto emocional de la epidemia de coronavirus en las enfermeras y otros profesionales de la salud. Enfermería Clínica [Internet]. 2020. [consultado el 27 de octubre de 2020]; mayo; 16. Disponible en: https://doi.org/10.1016/j.enfcli.2020.05.006 
5. Giacomello KJ, Melo LD. El significado del cuidado del niño hospitalizado: experiencias de profesionales de enfermería. Revista Brasileira de Enfermagem [Internet]. 2019. [consultado el 27 de octubre de 2020]; 72, 251-258. Disponible en: https://doi.org/10.1590/0034-7167-20180597.

6. Guerra N, Reina R, Cárdenas MH, Sanmiguel F. Significado del cuidado de la gestante desde la experiencia de la enfermera. Ágora Revista Científica [Internet]. 2019. [consultado el 27 de octubre de 2020]; 6(1):e4. Disponible en: http://dx.doi.org/10.21679/arc.v6i1.125

7. Ramírez EN. Significado, relevancia y elementos de género asociados al cuidado. Metasíntesis cualitativa. Index enferm [Internet]. 2016. [consultado el 27 de octubre de 2020]; 25(1-2):33-37. Disponible en: http://scielo.isciii.es/scielo.php?script=sci_artte $x t \& p i d=S 1132-12962016000100008 \&$ lng $=$ es.

8. Martínez AL. Entrevista a Enrique Dussel. Analectica [Internet]. 2015. [consultado el 27 de octubre de 2020]. 1(8). Disponible en: https://doi.org/10.5281/zenodo.3910794

9. Creswell J, Poth C. Qualitive inquiry and research design: choosing among five approaches. $4^{\text {th }}$. ed. EUA: SAGE. 2017.

10. Rodríguez-Bustamante P, Baez-Hernández, F. Epistemología de la profesión Enfermera. ENE de Enfermería [Internet]. 2020. [consultado el 27 de octubre de 2020]. 14(2). Disponible en: http://www.ene-

enfermeria.org/ojs/index.php/ENE/article/view/ 958

11. Errasti - Ibarrondo B, Jordán JA, Díez - Del Corral MP, Arantzamendi, M. Van Manen's phenomenology of practice: How can it contribute to nursing? Nursing Inquiry [Internet]. 2019. [consultado el 30 de noviembre de 2020]. 26(1), e12259. Disponible en: https://doi.org/10.1111/nin.12259

12. Hernández SR, Mendoza TC. Metodología de la investigación. Las Rutas cuantitativas, cualitativa y mixta. 2. a ed. España: McGraw Hill, Interamericana. 2018.

13. Glaser B, Strauss AL. The Discovery of Grounded Theory: Strategies for Qualitative Research. New York: Routledge. 2017.

14. Monistrol O. El trabajo de campo en la investigación cualitativa (II). Nure Investigación [Internet]. 2007. [consultado el 27 de octubre de 2020]. 29: 1-4. Disponible en: https://www.nureinvestigacion.es/OJS/index.ph $\mathrm{p} /$ nure/article/view/350

15. Azalte T, Puerta A, Morales R. Una mediación pedagógica en educación superior en salud. El diario de campo. Revista Iberoamericana de Educación [Internet]. 2008. [consultado el 27 de octubre de 2020]. 47(4):1-10. Disponible en: https://doi.org/10.35362/rie4742301

16. Robles B. La entrevista en profundidad: una técnica útil dentro del campo antropofísico. Red de Revistas científicas de América Latina, el Caribe, España y Portugal (Cuicuilco). [Internet]. 2011. [consultado el 27 de octubre de 2020]. 18(42): $40 . \quad$ Disponible en: http://www.scielo.org.mx/scielo.php?script=sci_ arttext\&pid=S0185-

$16592011000300004 \& \mid \mathrm{ng}=\mathrm{es} \& \mathrm{nrm}=\mathrm{iso} \& \mathrm{tlng}=$ es

17. Speziale HS, Streubert HJ, Carpenter DR. Qualitative research in nursing: advancing the humanistic imperative. 5th ed. Philadelphia, London: Lippincott Williams \& Wilkins; 2011.

18. Berguera $O$, Fernandez de Sanmamed $S$, Pons V, Pujol R, Rodríguez A, Saura S. Escuchar, observar y comprender. Recuperando la narrativa en las ciencias de la salud. Aportaciones de la investigación cualitativa. [Internet]. 2014 [consultado Junio 2020]; Disponible en: https://saludcomunitaria.files.wordpress.com/2 014/12/escucharobservarcomprender.pdf

19. Secretaria de Salud (SS). Reglamento de la Ley General de Salud. [Internet]. [consultado el 27 de octubre de 2020]. Disponible en: 
http://www.salud.gob.mx/unidades/cdi/nom/c ompi/rlgsmis.html

20. Peter R. Una visión Humana de los Humano. 1 ra ed. México, Puebla: Benemérita Universidad Autónoma de Puebla. 2006.

21. Sun $N$, Wei $L$, Shi $S$, Jiao $D$, Song $R, M a ~ L$, Wang $H$, Wang $C$, Wang $Z$, You Y, Liu S, Wang $H$. A qualitative study on the psychological experience of caregivers of COVID-19 patients. American journal of infection control [Internet]. 2020. [consultado el 27 de octubre de 2020]. 48(6): 592-598. Disponible en: https://doi.org/10.1016/j.ajic.2020.03.018

22. Maturana $H$, Bernard P. Del ser al hacer. Los orígenes de la biología del conocer. Buenos Aires: Granica; 2010.

23. Carper B. Fundamental patterns of knowing in nursing. Advances in nursing science [Internet]. 1978. [consultado el 27 de octubre de 2020]. 1(1):13-24. Disponible en: https://bit.ly/2FEFqmm

24. Boykin A, Schoenhofer A. Nursing as caring: a model for transforming practice. Createspace Independent Publishing Platform. United States of America. 2018.

25. Fawcett J. Applying Conceptual Models of Nursing: Quality Improvement, Research, and Practice. Springer Publishing Company; 2016.

26. Watson J. Unitary Caring Science: Philosophy and Praxis of Nursing. University Press of Colorado; 2018.

27. Roy, C. Key issues in nursing theory: Developments, challenges, and future directions. Nursing research [Internet] 2018 [consultado el 30 de noviembre de 2020]. 67(2), 81-92. Disponible en: DOI: 10.1097 / NNR.0000000000000266

28. Arvea D. Palabra y trascendencia. Manual de la educación y alfabetización popular. Oaxaca, México: Editorial la Mano; 2008.
29. Swanson KM. Nursing as informed caring for the well being of others. The journal of nursing scholarship [Internet]. 1993. [consultado el 27 de octubre de 2020]. 24(4): 352-357. Disponible en: https://doi.org/10.1111/j.15475069.1993.tb00271.x

30. Boff L. Cuidar de sí y de los demás en tiempos de pandemia. Argentina. Editorial Universidad Nacional de Córdoba. 2020.

Copyright (C) 2020 SANUS Artículo de acceso abierto distribuido bajo Licencia Creative Commons 